\title{
Búsqueda de complejos de Fe con potencial actividad vía el Efecto caballo de Troya en Escherichia coli
}

\author{
Jesus Antonio Alvarado-Huayhuaz ${ }^{1,2}$, Reneé Isabel Huamán Quispe ${ }^{1}$, Kathleen Vanessa Miguel-Calderón ${ }^{1}$, \\ Paloma Nancy Carrascal-Márquez ${ }^{1}$, Ana Cecilia Valderrama Negrón ${ }^{1}$ \\ ${ }^{1}$ Laboratorio de Investigación en Biopolímeros y Metalofármacos (LIBIPMET), Universidad Nacional de \\ Ingeniería, Lima, Perú \\ ${ }^{2}$ Grupo de Química Computacional Aplicada, Instituto de Química, Universidad de Sao Paulo, Sao Paulo, \\ Brasil
}

Enviado el 21 de diciembre del 2021. Aceptado el 9 de enero del 2022.

DOI: https://doi.org/10.33017/RevECIPeru2021.0013/

\section{Resumen}

La resistencia de microorganismos patógenos a los antimicrobianos es un problema mundial. Nuevas estrategias químicas como el efecto Caballo de Troya mediada por sideróforos representan una alternativa viable para esta problemática. Los microorganismos sintetizan sideróforos para captar hierro del medio extracelular e internalizarlo a través de proteínas de membrana externa, como por ejemplo la proteína FhuA en Escherichia coli. La desferrioxamina es un sideróforo producido por Streptomyces pilosus, pero el complejo hierro-desferrioxamina (FeDFO) puede ser asimilado también por E. coli. La promiscuidad, en torno al uso de sideróforos, justifica la búsqueda de nuevos complejos de hierro con potencial afinidad por FhuA. En este trabajo empleamos una nueva base de datos conteniendo 86665 compuestos de coordinación (tmQM). Mediante un análisis exploratorio de datos de tmQM y docking molecular se obtuvieron 4 complejos con mayor reactividad química y afinidad por FhuA que FeDFO. Interacciones hidrofóbicas y enlaces puente de hidrógeno son predominantes en el reconocimiento de estos complejos.

Descriptores: Complejos de hierro, desferrioxamina, docking molecular, FhuA, efecto Caballo de Troya

\section{Abstract}

Antimicrobial resistance of pathogens is a global problem. New chemical strategies such as the Trojan Horse effect mediated by siderophores represent a viable alternative to this problem. Microorganisms synthesize siderophores to scavenge iron from the extracellular medium and internalize it through outer membrane proteins, such as FhuA protein in Escherichia coli. Deferoxamine is a siderophore produced by Streptomyces pilosus, but iron-deferoxamine complex (FeDFO) can also be assimilated by $E$. coli. Promiscuity around the use of siderophores justifies the search for new iron complexes with potential affinity for FhuA. In this work we use a new database containing 86665 coordination compounds (tmQM). By means of an exploratory analysis of tmQM data and molecular docking, 4 complexes with higher chemical reactivity and affinity for FhuA than FeDFO were obtained. Hydrophobic and hydrogen bond interactions are predominant in the recognition of these complexes.

Keywords: Iron complexes, Deferoxamine, molecular docking, FhuA, Trojan horse effect

\section{Introducción}

La resistencia de patógenos a los antimicrobianos (AMR, por sus siglas en inglés: Antimicrobial Resistance) surge cuando estos agentes, como bacterias, virus, hongos o parásitos dejan de responder a los medicamentos, disminuyendo su ineficacia y provocando infecciones más difíciles de tratar o hasta imposibles. La AMR es hoy una problemática mundial que impacta principalmente 
en seguridad alimentaria, comercio internacional y desarrollo económico, obstaculizando directamente los Objetivos de Desarrollo Sostenible, como reporta la Organización Mundial de la Salud (ONU). Esta amenaza fue advertida desde el inicio del desarrollo de los primeros antimicrobianos por Alexander Fleming [1], sin embargo, recién en la década anterior se están tomando medidas sanitarias a nivel de jefes de estado y organizaciones mundiales directamente involucradas [2].

El "Efecto Caballo de Troya" (THA, por sus siglas en inglés: Trojan Horse Approach) es una estrategia química que consiste en la promoción de un agente tóxico (antibiótico, antiviral, antifúngico o antiparasitario) hacia el patógeno, empleando mecanismos biológicos propios de este [3]. THA alude a la historia de la Guerra de Troya y actualmente representa una alternativa viable frente a la resistencia antibacterial [4]. Los sideróforos son moléculas biosintetizadas por casi todos los microorganismos. Estas moléculas presentan grupos quelantes principalmente de tipo hidroxamato, catecolato o $\alpha$-hidroxicarboxilatos, que favorecen la formación de complejos de hierro con alta estabilidad [5]. Microorganismos como Pseudomonas, Mycobacterium, Enterobactinas, entre otros, se han vuelto resistentes a diversos antibióticos y presentan una membrana externa con baja permeabilidad a los fármacos, por ello, las proteínas receptoras de hierro-sideróforos en su membrana externa representan un potencial canal de entrada para nuevos candidatos a fármacos via THA [6]-[8]. La desferrioxamina B (DFO) es un sideróforo sintetizado por Streptomyces pilosus. Coordinado a hierro (FeDFO) puede ser asimilado por diversos microorganismos como Pseudomonas aeruginosa, Staphylococcus aureus, Escherichia coli, entre otros [9]. Tal promiscuidad en torno al uso de sideróforos representa una gran oportunidad en síntesis química y desarrollo de fármacos, sin embargo, estas investigaciones requieren equipos de caracterización, reactivos y solventes químicos, personal capacitado, entre otros factores que limitan su accesibilidad. Las simulaciones computacionales son una herramienta de amplia aplicación en el proceso de desarrollo de descubrimiento de fármacos, siendo el docking molecular una de las herramientas más destacadas debido a que nos permite buscar las poses ligando-receptor más afines (energía de afinidad o docking score más negativas) y explorar las interacciones intermoleculares que la componen [10]-[12]. El uso de bases de datos puede también optimizar este proceso de búsqueda disminuyendo costos y facilitando el acceso a investigaciones de alto impacto. En este trabajo se realizó exitosamente la búsqueda de complejos de hierro más afines por la proteína receptora de membrana externa FhuA de E. coli (receptor apto para el reconocimiento de hierro-sideróforo del tipo hidroxamato), empleando una nueva base de datos de compuestos de coordinación (tmQM), basada en complejos mononucleares principalmente de Cambridge Structural Database, que provee información de coordenadas cartesianas y propiedades de 86665 complejos singletes de capa cerrada [13].

\section{Metodología}

Se obtuvo la base de datos tmQM, con licencia Creative Commons Attribution 4.0 International, de github.com/bbskjelstad/tmqm. Mediante el uso de Notepad++, Jupyter Notebook y Python 3.8, se obtuvieron todos los complejos de hierro (4446) en formato $X Y Z$ y una tabla recopilatoria de sus propiedades disponible libremente en github.com/inefable12/tmQM_num_csd. Open Babel fue empleado para la conversión al formato SDF. Herramientas gratuitas en la nube (Google Colaboratory, Pandas, Numpy, Matplotlib, Statsmodels y Seaborn) fueron empleadas en el análisis exploratorio de datos (EDA). De acuerdo con el EDA se seleccionaron 100 compuestos para acoplarse con FhuA. El programa Maestro y distintos módulos de la suite Schrodinger 2021-3 (Glide, Macromodel, Ligand Interaction Diagram), son ampliamente usados en el desarrollo de docking molecular de compuestos de coordinación [14]-[17]. El receptor FhuA fue obtenido de la base de datos PDB RCSB, con el identificador 4CU4 [18], se eliminaron las moléculas de agua, ligando cocristalizado y otros componentes distintos a los aminoácidos. Se empleó Protein Preparation Wizard y MolProbity [19], para optimizar FhuA con el campo de fuerza OPLS3 y validarla vía z-score, respectivamente. Se asignaron los estados de protonación a pH 7.4 en el receptor empleando PropKa. La generación de la malla de búsqueda y la caja de cálculo (30 Å de arista) fueron realizadas con el módulo Receptor Grid Generator. Se evaluó previamente la carga formal, número de enlaces covalentes y reconocimiento de enlaces dativos en los complejos de hierro, siendo descartados aquellos con inconsistencias en carga o átomos faltantes de acuerdo con la información provista en la base de datos. Se generaron 10 poses por metalligando, considerándose la de mayor afinidad para el análisis de interacciones intermoleculares. 


\section{Resultados y Discusión}

Ocho variables en unidades atómicas, excepto el momento dipolar (Dipole_M), en Debye, son exploradas mediante diagramas de violín (Figura 1): Energía electrónica (Electronic_E), Dispersión (Dispersion_E), Momento dipolar (Dipole_M), Carga del metal (Metal_q), energía HOMO (HOMO_Energy), energía LUMO (LUMO_Energy), energía brecha, (HL_Gap) y polarizabilidad (Polarizability). Únicamente Metal_q, en azul, presenta dos regiones más pobladas, debido a que los complejos presentan hierro en más de un estado de oxidación. Por otro lado, se observa una distribución normal respecto de la mediana y no se observan valores atípicos.

FeDFO es asimilado por $E$. coli mediante un acoplamiento inicial en FhuA, por ello, podemos tomar este sistema FeDFO-FhuA como un control positivo para el reconocimiento de complejos de hierro. Este complejo contiene un átomo de hierro en estado de oxidación 3+, hexacoordinado por 3 grupos hidroxamatos con isomería fac, una brecha energética entre HOMO y LUMO alrededor de $3 \mathrm{eV}$ y un momento dipolar próximo de $35 \mathrm{D}$ [20]-[22]. Asociando esta información con la base de datos, nos enfocamos en disponer de compuestos con alta carga en el metal, alta polarizabilidad y baja energía brecha o alta reactividad química. Basándonos en la evaluación del coeficiente de correlación de Pearson $(\rho)$, podemos señalar una fuerte relación positiva en valores cercanos $a+1$, fuerte relación negativa, en valores próximos a -1 , o ninguna relación en valores próximos a cero. De acuerdo con la matriz de correlación (Figura 2), identificamos una correlación negativa entre HL_Gap y Metal_q: $\rho=-0.71$, y en menor grado para $\bar{H} L$ Gap frente a polarizabilidad $(\rho=-0.31)$ y energía $\operatorname{HOMO}(\rho=-$ 0.47).
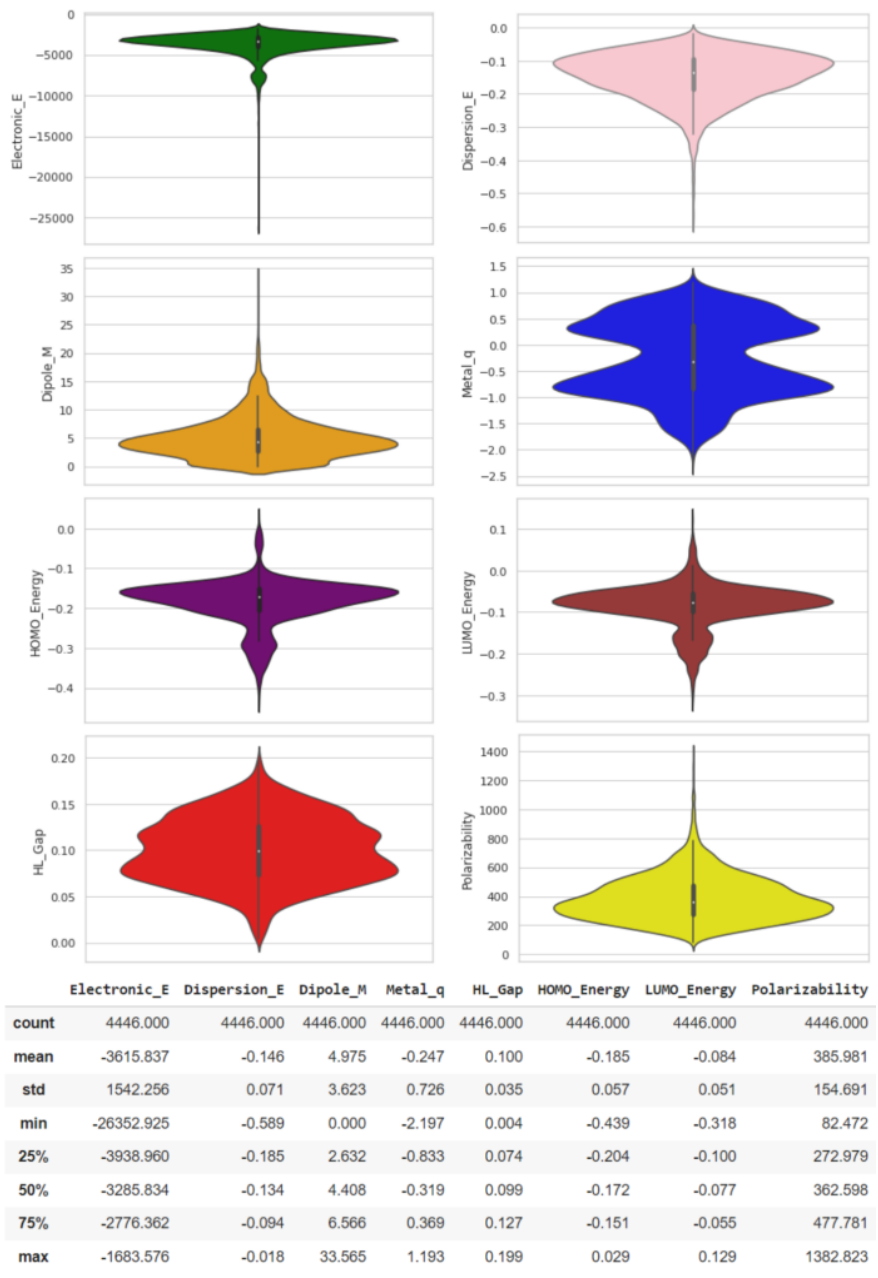

Figura 1: Diagramas de violín de las variables reportadas en tmQM para los complejos de hierro.

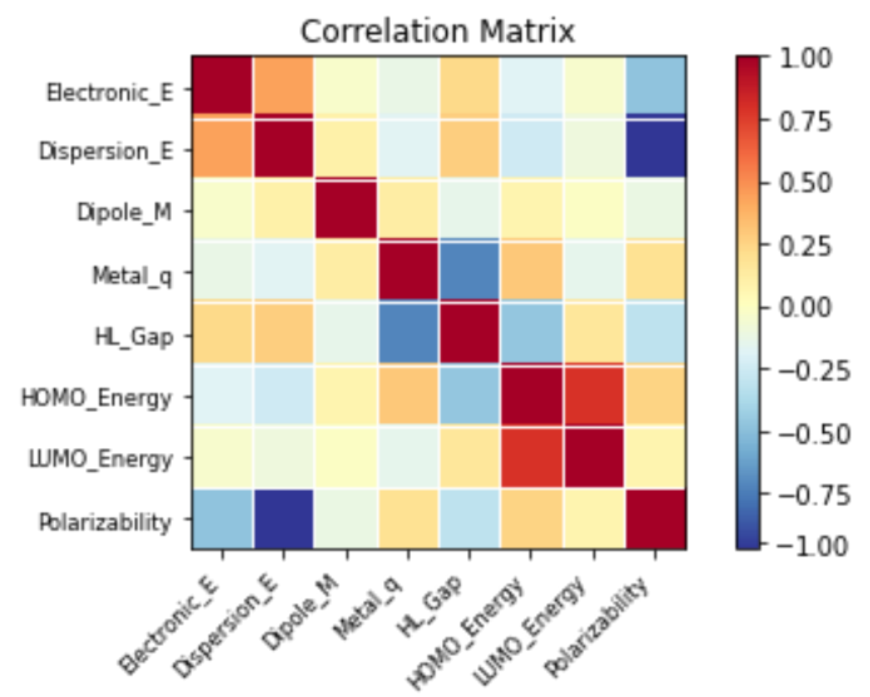

Figura 2: Matriz de correlación entre las variables en tmQM para los complejos de hierro.

Tomando en cuenta los criterios mencionados seleccionamos los primeros 100 complejos de 
Revista ECIPerú

hierro (disponible libremente en github.com/inefable12/tmQM_num_csd/blob/main/ Fe_complx_filtrado_ordenado_x_HLGap_200.csv). FeDFO se acopló en FhuA con $-5.47 \overline{\mathrm{kcal}} / \mathrm{mol}$ (Figura 3), estabilizándose principalmente con residuos hidrofóbicos (círculos verdes).

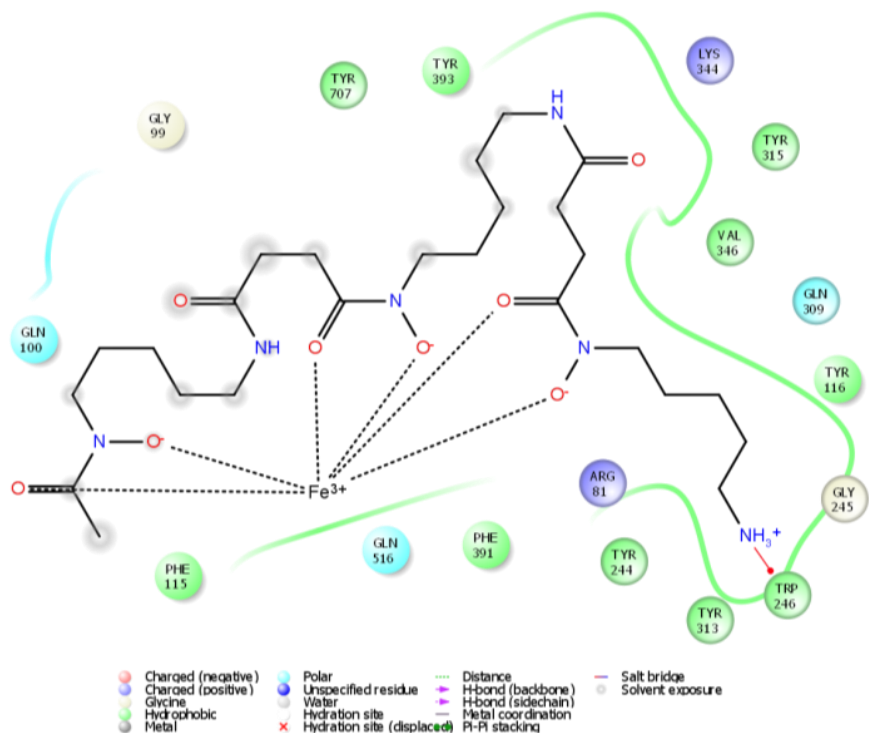

Figura 3: Diagrama de interacción 2D de FeDFO en FhuA. Docking score: $-5.479 \mathrm{kcal} / \mathrm{mol}$.

4 de los 100 primeros complejos con la menor HL_Gap de tmQM, se acoplaron con docking score menores a $-7 \mathrm{kcal} / \mathrm{mol}$, es decir, presentan mayor afinidad por FhuA que FeDFO. El acoplamiento de FORVUE en FhuA es el de mayor afinidad de toda la base de datos, $-7.496 \mathrm{kcal} / \mathrm{mol}$, con hasta 4 enlaces puente de hidrógeno (Figura 4). Los otros mejores acoplamientos se dieron con HOPJAY (Figura 5), UGENOK (Figura 6) y CEVXOT (Figura 7). En estos diagramas podemos observar también la predominancia de aminoácidos hidrofóbicos, como en el caso de FeDFO, e interacciones intermoleculares producidas principalmente por enlaces puente de hidrógeno.
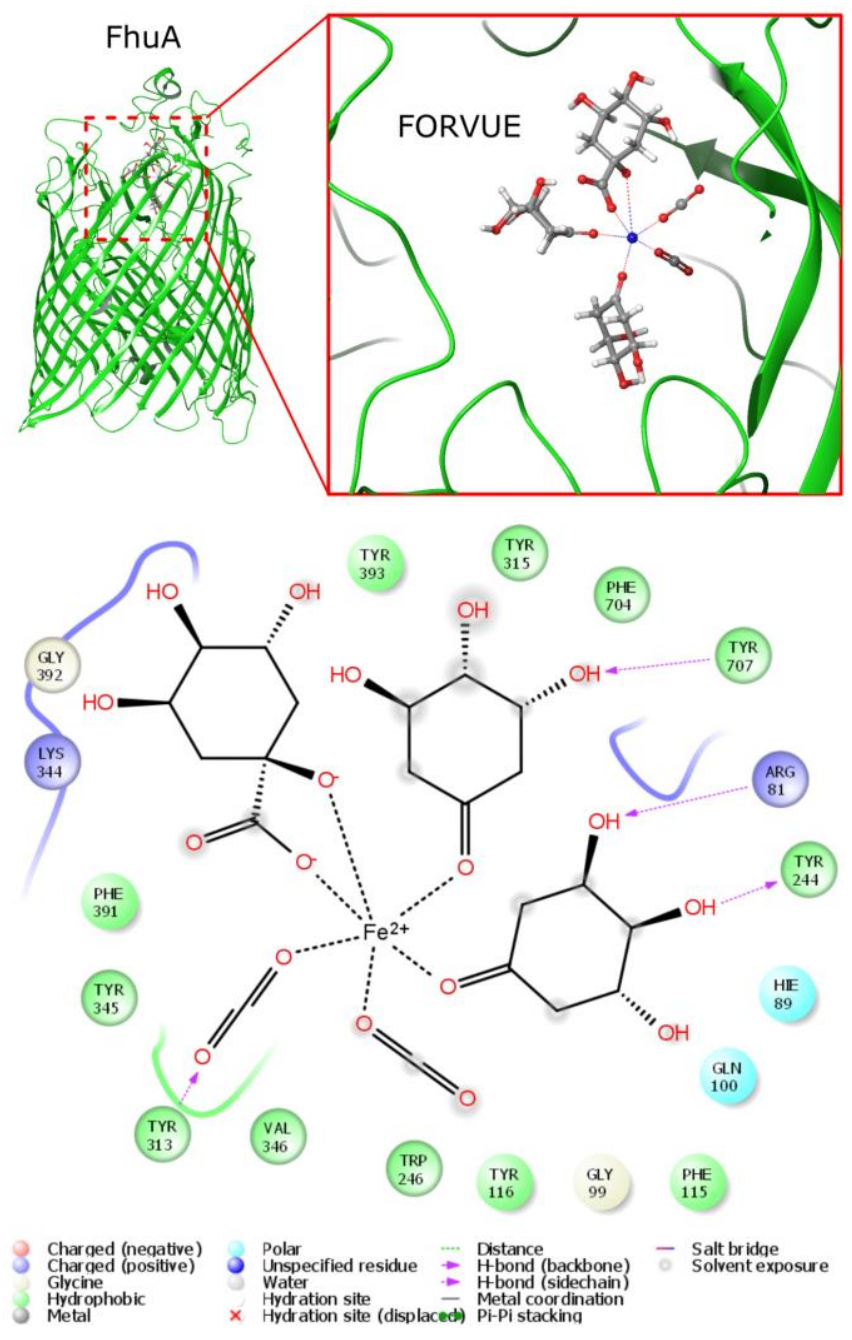

Figura 4: Visualización $3 D$ del acoplamiento FORVUE-FhuA y diagrama de interacción $2 D$. Docking score: $-7.946 \mathrm{kcal} / \mathrm{mol}$.

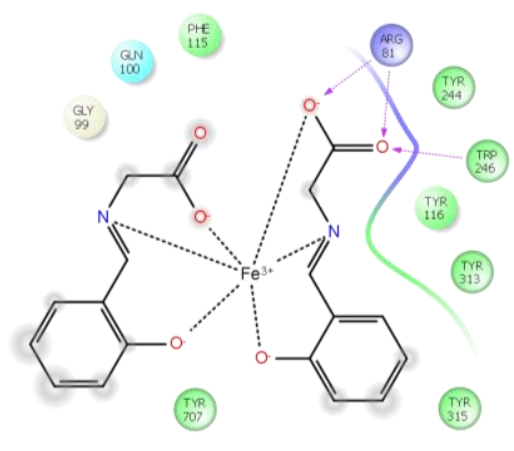

Figura 5: Diagrama de interacción 2D de UGENOK en FhuA. Docking score: $-7.878 \mathrm{kcal} / \mathrm{mol}$. 


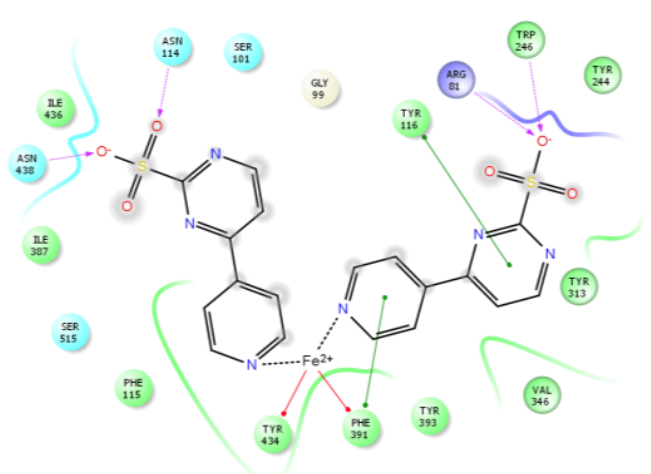

Figura 6: Diagrama de interacción 2D de HOPJAY en FhuA . Docking score: $-7.591 \mathrm{kcal} / \mathrm{mol}$.

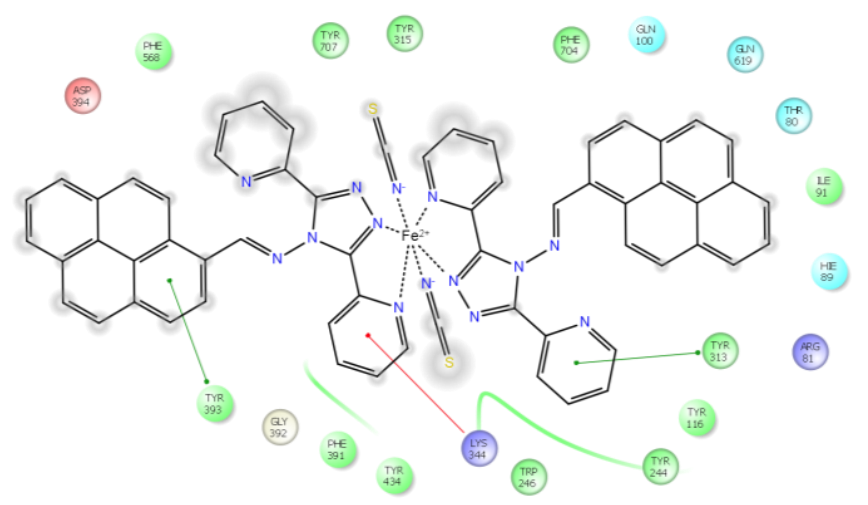

Figura 7: Diagrama de interacción 2D de CEVXOT en FhuA. Docking score: $-7.278 \mathrm{kcal} / \mathrm{mol}$.

La afinidad energética de FeDFO en FhuA, se debe principalmente a aportes energéticos por interacciones intermoleculares lipofílicas, pi-catión y en menor grado electrostáticas (Figura 8). Si bien los complejos FORVUE, HOPJAY, UGENOK y CEVXOT presentan mayor afinidad que FeDFO, estos no registran las mismas interacciones intermoleculares con FhuA, por lo cual, no es de esperarse un mecanismo de acción biomimético en $E$. coli, sin embargo, la elevada afinidad de estos 4 complejos muestra su potencial actividad vía THA [23]-[25]. Como perspectiva futura se puede evaluar la evolución en el tiempo de estos acoplamientos mediante simulaciones de dinámica molecular, el uso de inteligencia artificial para el reconocimiento de patrones en las interacciones intermoleculares encontradas, así como también, estudios in vitro para la evaluación de la dosisrespuesta en $E$. coli.

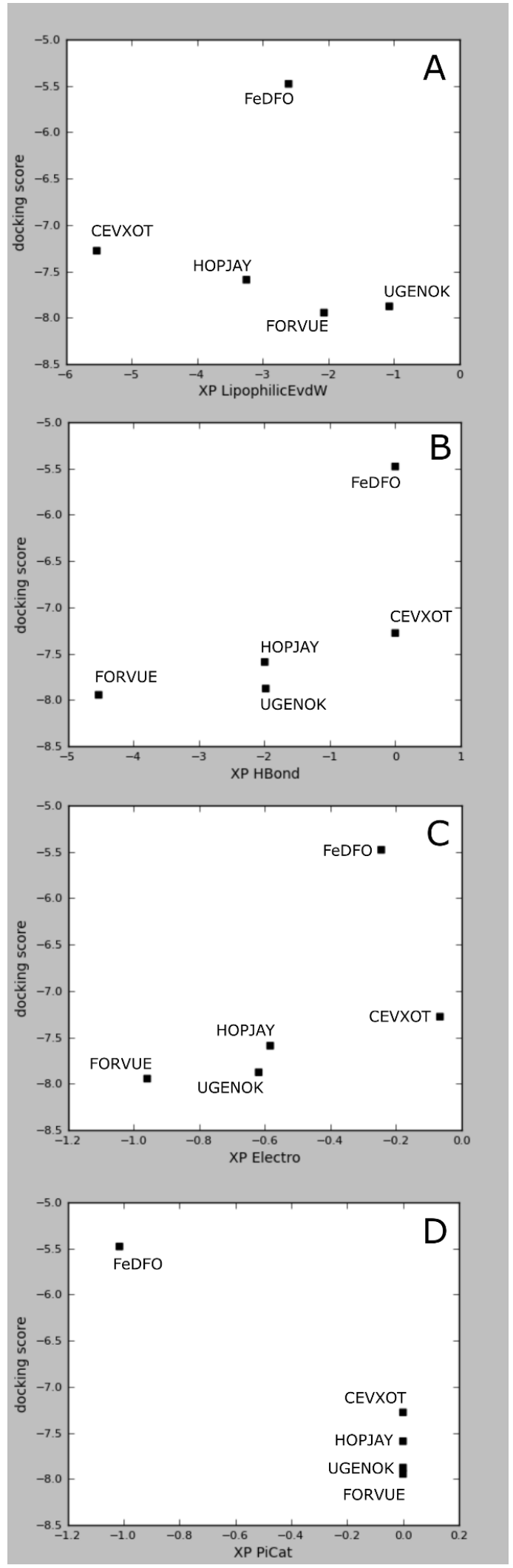

Figura 8: Docking score respecto de: A. Lipofilia, $B$. enlace puente de hidrógeno, $C$. interacciones electrostáticas y D. Pi-catión.

Por otro lado, destacamos también otros 12 compuestos que superaron a FeDFO en afinidad con FhuA, en el rango de -7 a $-6 \mathrm{kcal} / \mathrm{mol}$ : CARQUH 
(-6.598), XANMIK (-6.305), AHOHIP (-6.194), TUNKET (-6.177), HAYBEQ (-6.046), BAZCIQ (6.912), HENLIV (-6.419), OXUFAQ (-6.652), MELBOU (-6.609), SUMWUS (-6.381), JUPRAN (6.112 ) y NOWBIK (-6.079). Así, en total reportamos 16 complejos de hierro obtenidos a partir de 86665 en tmQM con mayor reactividad química que FeDFO y mayor afinidad por su proteína receptora en la membrana de $E$. coli.

\section{Conclusiones}

Encontramos y analizamos 4 complejos de hierro (con código CSD: FORVUE, HOPJAY, UGENOK y CEVXOT) entre 86665 compuestos metal-ligando mononucleares provenientes principalmente de Cambridge Structural Database, contenidos en una reciente base de datos, tmQM. Estos complejos, de mayor reactividad química que FeDFO, presentaron mayor afinidad por la proteína de membrana externa FhuA, encargada de la identificación e internalización de sus complejos hierro-sideróforos. Ante la necesidad de vías farmacológicas alternativas, debido a la resistencia de patógenos a los antibióticos, nuestro estudio pone en relevancia un enfoque de búsqueda rápida y eficaz de potenciales nuevos candidatos a actuar mediante THA.

\section{Agradecimientos}

JAAH agradece a FONDECYT (Convenio 2372015-FONDECYT) por la concesión de la beca de doctorado.

\section{Referencias}

[1] R. Gaynes, "The Discovery of PenicillinNew Insights After More Than 75 Years of Clinical Use," Emerg. Infect. Dis., vol. 23, no. 5, pp. 849-853, May 2017.

[2] Interagency Coordination Group on Antimicrobial Resistance, "No Time to Wait: Securing the future from drug-resistant infections," 2019. [Online]. Available: https://www.who.int/antimicrobialresistance/interagency-coordinationgroup/final-report/en/. [Accessed: 25-Feb2021].

[3] K. N. Raymond, B. E. Allred, and A. K. Sia, "Coordination Chemistry of Microbial Iron Transport," Acc. Chem. Res., vol. 48, no. 9, pp. 2496-2505, Sep. 2015.

[4] Negash, Norris, and Hodgkinson, "Siderophore-Antibiotic Conjugate Design:
New Drugs for Bad Bugs?," Molecules, vol. 24, no. 18, p. 3314, Sep. 2019.

[5] D. J. Raines, T. J. Sanderson, E. J. Wilde, and A.-K. Duhme-Klair, "Siderophores," in Reference Module in Chemistry, Molecular Sciences and Chemical Engineering, Elsevier, 2015.

[6] A. Pandey, C. Savino, S. H. Ahn, Z. Yang, S. G. Van Lanen, and E. Boros, "Theranostic Gallium Siderophore Ciprofloxacin Conjugate with Broad Spectrum Antibiotic Potency," J. Med. Chem., vol. 62, no. 21, pp. 9947-9960, Nov. 2019.

[7] V. Antonietti et al., "Synthesis, iron(III) complexation properties, molecular dynamics simulations and $P$. aeruginosa siderophore-like activity of two pyoverdine analogs," Eur. J. Med. Chem., vol. 137, pp. 338-350, Sep. 2017.

[8] C. C. C. R. de Carvalho and P. Fernandes, "Siderophores as 'Trojan Horses': tackling multidrug resistance?," Front. Microbiol., vol. 5, Jun. 2014.

[9] J. A. A. Huayhuaz, H. A. Vitorino, O. S. Campos, S. H. P. Serrano, T. M. Kaneko, and B. P. Espósito, "Desferrioxamine and desferrioxamine-caffeine as carriers of aluminum and gallium to microbes via the Trojan Horse Effect," J. Trace Elem. Med. Biol., vol. 41, pp. 16-22, May 2017.

[10] F. D. Prieto-Martínez and J. MedinaFranco, "Diseno de fármacos asistido por computadora: cuando la informática, la química y el arte se encuentran," TIP. Rev. Espec. en Ciencias Químico-Biológicas, vol. 21, 2018.

[11] W. Filgueira de Azevedo, Docking Screens for Drug Discovery. Springer New York, 2020.

[12] P. H. M. Torres, A. C. R. Sodero, P. Jofily, and F. P. Silva-Jr, "Key Topics in Molecular Docking for Drug Design," Int. J. Mol. Sci., vol. 20, no. 18, p. 4574, Sep. 2019.

[13] D. Balcells and B. B. Skjelstad, "tmQM Dataset-Quantum Geometries and Properties of $86 \mathrm{k}$ Transition Metal Complexes," J. Chem. Inf. Model., vol. 60, no. 12, pp. 6135-6146, Dec. 2020.

[14] R. A. Friesner et al., "Extra Precision Glide: Docking and Scoring Incorporating a Model of Hydrophobic Enclosure for Protein-Ligand Complexes," J. Med. Chem., vol. 49, no. 21, pp. 6177-6196, Oct. 2006. 
[15] H. S. N. Prasad et al., "Design, synthesis and molecular docking studies of novel piperazine metal complexes as potential antibacterial candidate against MRSA," J. Mol. Struct., vol. 1232, p. 130047, May 2021.

[16] P. Vijayakrishnan, S. Arul Antony, and D. Velmurugan, "Structural data of DNA binding and molecular docking studies of dihydropyrimidinone transition metal complexes," Data Br., vol. 19, pp. 817-825, Aug. 2018.

[17] A. Adeniyi and P. Ajibade, "Comparing the Suitability of Autodock, Gold and Glide for the Docking and Predicting the Possible Targets of $\mathrm{Ru}(\mathrm{II})$-Based Complexes as Anticancer Agents," Molecules, vol. 18, no. 4, pp. 3760-3778, Mar. 2013.

[18] I. Mathavan et al., "Structural basis for hijacking siderophore receptors by antimicrobial lasso peptides," Nat. Chem. Biol., vol. 10, no. 5, pp. 340-342, May 2014.

[19] C. J. Williams et al., "MolProbity: More and better reference data for improved all-atom structure validation," Protein Sci., vol. 27, no. 1, pp. 293-315, Jan. 2018.

[20] S. D. Domagal-Goldman, K. W. Paul, D. L. Sparks, and J. D. Kubicki, "Quantum chemical study of the $\mathrm{Fe}(\mathrm{III})$ desferrioxamine $\mathrm{B}$ siderophore complexElectronic structure, vibrational frequencies, and equilibrium Fe-isotope fractionation," Geochim. Cosmochim. Acta, vol. 73, no. 1, pp. 1-12, Jan. 2009.
[21] S. Dhungana, P. S. White, and A. L. Crumbliss, "Crystal structure of ferrioxamine B: a comparative analysis and implications for molecular recognition," JBIC J. Biol. Inorg. Chem., vol. 6, no. 8, pp. 810-818, Oct. 2001.

[22] D. Bellotti and M. Remelli, "Deferoxamine B: A Natural, Excellent and Versatile Metal Chelator," Molecules, vol. 26, no. 11, p. 3255, May 2021.

[23] L. Moynié et al., "The complex of ferricenterobactin with its transporter from Pseudomonas aeruginosa suggests a twosite model," Nat. Commun., vol. 10, no. 1, p. 3673, Dec. 2019.

[24] Z. Li, S. Chinnasamy, Y. Zhang, and D.-Q. Wei, "Molecular dynamics simulation and binding free energy calculations of microcin J25 binding to the FhuA receptor," J. Biomol. Struct. Dyn., pp. 1-10, Apr. 2020.

[25] I. Josts, K. Veith, and H. Tidow, "Ternary structure of the outer membrane transporter FoxA with resolved signalling domain provides insights into TonB-mediated siderophore uptake," Elife, vol. 8, Aug. 2019.

\section{E-mail:}

jalvaradoh@uni.pe, renee.huaman.q@uni.pe, kathleen.miguel.c@uni.pe, paloma.carrascal.m@uni.pe, ana.valderrama.n@uni.edu.pe 